\title{
Supersymmetric Dark Matter or Not
}

\author{
Keith A. Olive* \\ William I. Fine Theoretical Physics Institute, School of Physics and Astronomy, \\ University of Minnesota, Minneapolis, MN 55455, USA \\ E-mail: olive@umn. edu
}

\begin{abstract}
The lack of evidence for low energy supersymmetry at the LHC implies a supersymmetry scale in excess a TeV. While this is consistent (and even helpful) with a Higgs boson mass at $\approx 125 \mathrm{GeV}$, simple supersymmetric models with scalar and gaugino mass universality are being pushed into strips of parameter space. These often require coannihilations to obtain an acceptable relic density and the extent of these coannihilation strips will be discussed. In contrast, non-supersymmetric grand unified theories such as $\mathrm{SO}(10)$ may also provide a dark matter candidate. Because of the presence of an intermediate scale, these theories may unify gauge couplings, provide for neutrino masses and a suitably long lived proton.
\end{abstract}

The 11th International Workshop Dark Side of the Universe 2015

14-18 December 2015

Kyoto, Japan

${ }^{*}$ Speaker. 


\section{Before Run I}

Despite its relative simplicity as an extension of the Standard Model (SM), there are many possible realizations of low energy supersymmetry which can be traced to the unknown mechanism of supersymmetry breaking and the multitude of parameters associated with that breaking. The most widely studied version of low energy supersymmetry makes some strong assumptions concerning these supersymmetry breaking parameters. Namely, it assumes that all gaugino masses are universal at some input renormalization scale (usually taken to be the Grand Unification (GUT) scale at which gauge coupling unification occurs). It further assumes that all supersymmetry breaking scalar masses and trilinear terms are also universal at the same input scale. This constrained version of the minimal supersymmetric standard model (CMSSM) $[1,2,3,4]$ is a 4-parameter theory defined by the gaugino mass, $m_{1 / 2}$, the scalar mass, $m_{0}$, the trilinear mass term, $A_{0}$, and the ratio of the two Higgs vacuum expectation values, $\tan \beta$. In the CMSSM, one uses the conditions derived by the minimization of the Higgs potential after radiative electroweak symmetry breaking to solve for the Higgs mixing mass, $\mu$ and the bilinear mass term $B_{0}$ (or equivalently $\mu$ and the Higgs pseudoscalar mass, $m_{A}$ ) for fixed $\tan \beta$.

As is now well known, Run I of the LHC did not provide evidence for supersymmetry, and instead pushed supersymmetric mass scales to higher energies of order a TeV. At the higher mass scales, viable dark matter models rely on enhanced annihilations in order to obtain the correct relic density. This may due to either coannihlations [5], direct s-channel annihilations on a pole (the funnel region) [5] or in the focus point where the $\mu$ parameter is relatively small [6]. The coannihilation and funnel mechanisms require very special choices of the input parameters which cause near degeneracies among the sparticle masses. Thus regions of good relic density typically lie in strips in a $\left(m_{0}, m_{1 / 2}\right)$ plane (the focus point also forms a strip along the boundary where radiative symmetry breaking fails). The ultimate potential for the discovery of supersymmetry depends on the extent of these strips.

An alternative to supersymmetric dark matter is provided by $\mathrm{SO}(10)$ grand unification [7, 8]. The presence of an intermediate scale allows for the possibility of gauge coupling unification (without supersymmetry) $[8,9,10,11,12,13]$ and the breaking of the intermediate scale provides a natural origin for the see-saw mechanism for generating neutrino masses [14]. If the intermediate scale is broken by a 126-dimensional Higgs representation, the theory preserves a $Z_{2}$ symmetry [15] which can account for the stability of a new dark matter candidate [10, 11, 12, 13, 16, 17].

Prior to Run I of the LHC, there was considerable excitement about the prospect for discovering supersymmetry as supersymmetric models such as the CMSSM provided definite improvements to low energy precision phenomenology. This can be seen in the left panel of Fig. 1 which shows the results of mastercode $[18,20]$ - a frequentist Markov Chain Monte Carlo analysis of low energy experimental observables in the context of supersymmetry. The figure shows the color coded values of $\Delta \chi^{2}$ relative to the best fit point shown by the white dot at low $m_{1 / 2}$ and low $m_{0}$. Marginalization over $A_{0}$ and $\tan \beta$ was performed to produce this $\left(m_{0}, m_{1 / 2}\right)$ plane. The best-fit CMSSM point lies at $m_{0}=60 \mathrm{GeV}, m_{1 / 2}=310 \mathrm{GeV}, A_{0}=130 \mathrm{GeV}, \tan \beta=11$ yielding the overall $\chi^{2} / \mathrm{N}_{\text {dof }}=20.6 / 19$ (36\% probability) with $m_{h}=114.2 \mathrm{GeV}$. Recall that this was a pre-LHC prediction and uses no LHC data. Rather, it is based on a wide array of low energy observables including $\left(g_{\mu}-2\right), M_{W}, B \rightarrow \tau \nu, b \rightarrow s \gamma$, the LEP limit on the Higgs mass, forward-backward 
asymmetries among others (for a full list of observables used see [18]). The relatively low value of $m_{h}$ was a common prediction of MSSM models [21]. Indeed a dedicated scan for the distribution of Higgs masses in the CMSSM was made in [22] which found that when all phenomenological constraints (with or without $\left(g_{\mu}-2\right)$ ) are included, all models yielded $m_{h} \leq 128 \mathrm{GeV}$. When $\left(g_{\mu}-2\right)$ is included, only models with $m_{h}<126 \mathrm{GeV}$ were found. Note that the scan sampled scalar and gaugino masses only out to $2 \mathrm{TeV}$.
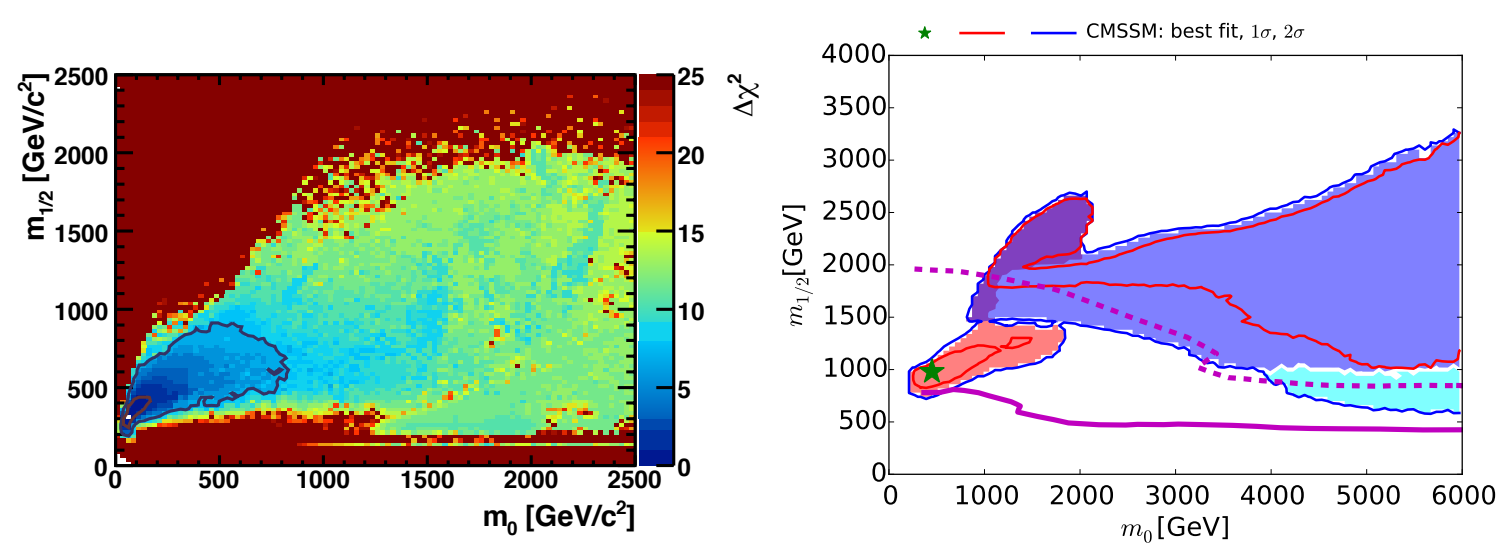

Figure 1: The $\Delta \chi^{2}$ functions in the $\left(m_{0}, m_{1 / 2}\right)$ planes for the CMSSM from a mastercode frequentist analysis. The pre-LHC result is shown in the left panel [18]. Red and blue contours correspond to 68\% and 95\% CL contours and the best fit point is depicted by a white dot. The post-LHC result is shown in the right panel [19] using $8 \mathrm{TeV}$ data at $20 \mathrm{fb}^{-1}$. Here the best fit point is shown by the filled star. The color of the shaded region indicates the dominant annihilation mechanism for obtained the correct relic density: stau coannihilation-pink; $A / H$ funnel-blue; focus point-cyan; and a hybrid region of stau coannihilation and funnel-purple. The solid and dashed purple curves show the run I reach and the expected run II reach at 14 $\mathrm{TeV}$ at $3000 \mathrm{fb}^{-1}$ respectively. The latter corresponds approximately to the $95 \% \mathrm{CL}$ exclusion sensitivity with $300 / \mathrm{fb}$ at $14 \mathrm{TeV}$.

This optimism of discovering supersymmetry spread to the prospects of discovering dark matter in direct detection experiments. The left panel of Fig. 2 displays the pre-LHC preferred range of the spin-independent DM scattering cross section $\sigma_{p}^{\text {SI }}$ (calculated here assuming an optimistic $\pi$-N scattering term $\Sigma_{N}=64 \mathrm{MeV}$ ) as a function of $m_{\chi}$ [18]. We see that the expected range of $\sigma_{p}^{\text {SI }}$ lies just below the then present experimental upper limits (solid lines) [23, 24]. As one can see from the successive lower upper limits from later experiments [25, 26, 27] shown by the bands, these pre-LHC values for the elastic scattering cross section showed great promise for discovery.

\section{After Run I}

As Run I at the LHC, progressed, it was becoming increasingly clear that supersymmetry if present at low energy at all, was at mass scales larger than originally anticipated [31]. Subsequent to the final run at the LHC, the picture looked very different. In the right panel of Fig. 1, the postRun I likelihood contours in the $\left(m_{0}, m_{1 / 2}\right)$ plane [19] are shown using $8 \mathrm{TeV}$ results at $20 \mathrm{fb}^{-1}$ [32]. The best fit point based on the $8 \mathrm{TeV}$ data is shown by the filled star at $(420,970) \mathrm{GeV}$ with $A_{0}=3000 \mathrm{GeV}$ and $\tan \beta=14$, though the likelihood function is quite flat and the exact position of the best point is not particularly meaningful. The $\chi^{2} / N_{\text {dof }}$ is now increased to $35.1 / 23(5.1 \%$ 

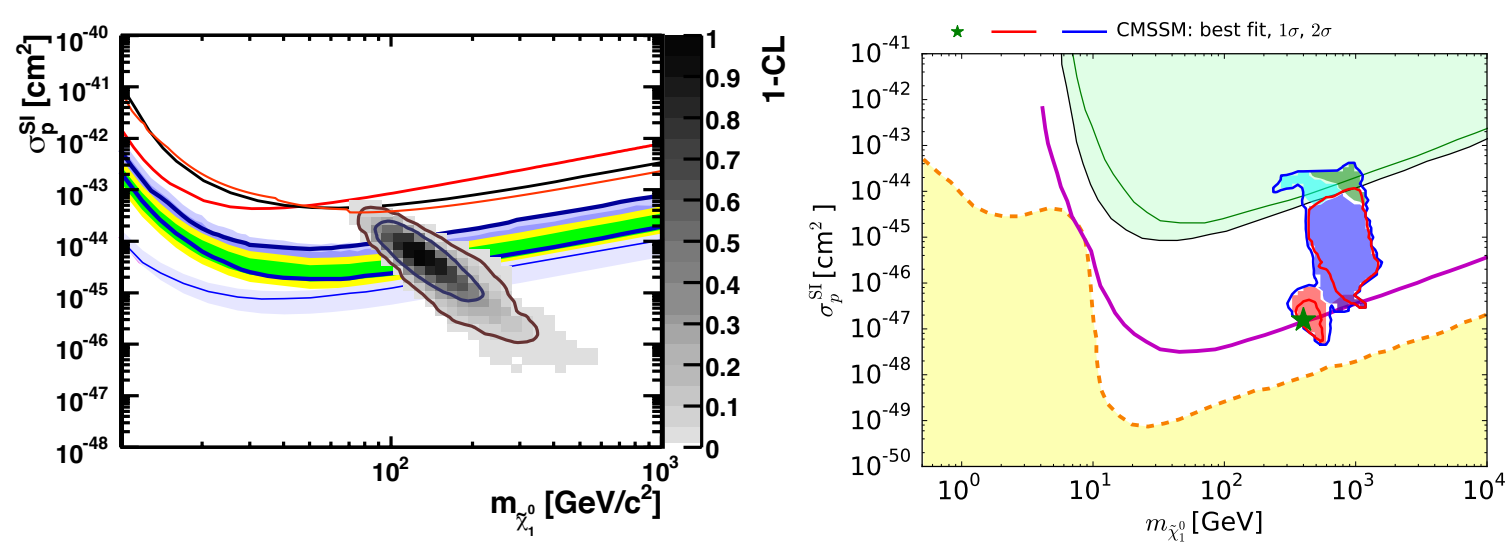

Figure 2: (left) The pre-LHC prediction for the spin-independent DM scattering cross section, $\sigma_{p}^{\mathrm{SI}}$, versus $m_{\chi}$ in the CMSSM [18]. The solid lines are the pre-LHC experimental upper limits from CDMS [23] and XENON10[24], while the bands are the more recent limits from XENON100 [25, 26] and LUX [27]. (right) The post-run I likelihood contours for $\sigma_{p}^{\text {SI }}$ [19]. Shading within the likelihood contours is the same as in Fig. 1, though here we also see a region where chargino coannihilations are dominant (green). The green and black lines show the current sensitivities of the XENON100 [26] and LUX [27] experiments, respectively, and the solid purple lines show the projected 95\% exclusion sensitivity of the LUX-Zepelin (LZ) experiment [28]. The dashed orange line shows the astrophysical neutrino 'floor' [29, 30], below which astrophysical neutrino backgrounds dominate (yellow region).

probability). This result may be compared with the Standard Model fit which yields $\chi^{2} / N_{\text {dof }}$ of $36.5 / 24$ (5.0\% probability), which of course ignores the fact that there is no dark matter candidate in the Standard Model.

We see in the right panel of Fig. 1 that three DM mechanisms dominate in the CMSSM: $\tilde{\tau}_{1}$ coannihilation at low $m_{0} \lesssim 2000 \mathrm{GeV}$, the $H / A$ funnel at larger $m_{0}$ and $m_{1 / 2}$, and the focus point at larger $m_{0}$ and smaller $m_{1 / 2}$ where the neutralino becomes a 'well-tempered' mixture of bino and Higgsino [33]. There is also a hybrid $\tilde{\tau}_{1} / A / H$ region extending up to $\left(m_{0}, m_{1 / 2}\right) \sim$ $(2000,2500) \mathrm{GeV}$.

As one can see from the right panel of Fig. 2, there is still hope for direct detection experiments though the new best fit point implies a cross section of $\sim 10^{-47} \mathrm{~cm}^{2}$, nearly two orders of magnitude below the current upper bound. As commented on previously, the likelihood function is rather flat between $10^{-47} \mathrm{~cm}^{2} \lesssim \sigma_{p}^{\mathrm{SI}} \lesssim 10^{-45} \mathrm{~cm}^{2}$. Note that in this case, we have adopted $\Sigma_{\pi N}=50 \pm 7$ $\mathrm{MeV}$. In addition to the model results, the $90 \% \mathrm{CL}$ upper limits on $\sigma_{p}^{\mathrm{SI}}$ given by the XENON100 and LUX experiments [26, 27] as well as the expected reach from LZ [28] are also displayed. The level of the atmospheric neutrino background [29, 30] is shown by the shaded region at small cross sections. The current XENON100 and LUX data already put strong pressure on models where the focus-point or chargino coannihilation mechanism dominates. There are borderline regions that are formally excluded by the $\sigma_{p}^{\text {SI }}$ data considered in isolation, but become permitted at the 95\% CL in a global fit including other observables, and also due to the uncertainties in the calculation of $\sigma_{p}^{\text {SI }}$ that have been included in the evaluation of the global $\chi^{2}$ function [34]. We also see that the $\tilde{\chi}_{1}^{ \pm}$ coannihilation region and most of the $H / A$ funnel region would be accessible to the planned LZ experiment. However, much of the $\tilde{\tau}_{1}$ coannihilation region lies below the LZ sensitivity, though it 
could be accessible to a 20-tonne DM experiment such as Darwin [35].

\section{The Strips}

As noted earlier, regions of parameter space with the correct relic density typically occur in relatively narrow strips formed by special relations among the input parameters. In these cases, the relic density is strongly affected by coannihilations, or rapid $s$-channel annihilations.

The stau coannihilation strip $[36,37]$ is present when the mass of the lighter stau is nearly degenerate with the lightest supersymmetric particle (LSP) which is often the bino in CMSSM models. An example (barely) showing the stau coannihilation strip is found in Fig. 3 which shows the $\left(m_{0}, m_{1 / 2}\right)$ plane for fixed $\tan \beta=20$ and $A_{0}=2.3 m_{0}$. In the dark red shaded region at small $m_{0}$ extending to large $m_{1 / 2}$, the lighter stau is the LSP and that region is excluded. Along the border of that region, the stau and lightest neutralino are degenerate. The stau coannihilation strip tracks that boundary up to roughly $m_{1 / 2}=1 \mathrm{TeV}$ and is shown as a blue shaded strip. Along the strip, the Higgs mass (shown by the red dot dashed curves computed with FeynHiggs [38]) does not exceed $124 \mathrm{GeV}$. The current and future reach of the LHC is shown by the solid black, blue, green and purple lines which are particle exclusion reaches for $\phi_{T}$ searches with $20 / \mathrm{fb}$ at $8 \mathrm{TeV}, 300$ and 3000/fb at $14 \mathrm{TeV}$, and 3000/fb at a prospective HE-LHC at $33 \mathrm{TeV}$, respectively [39].
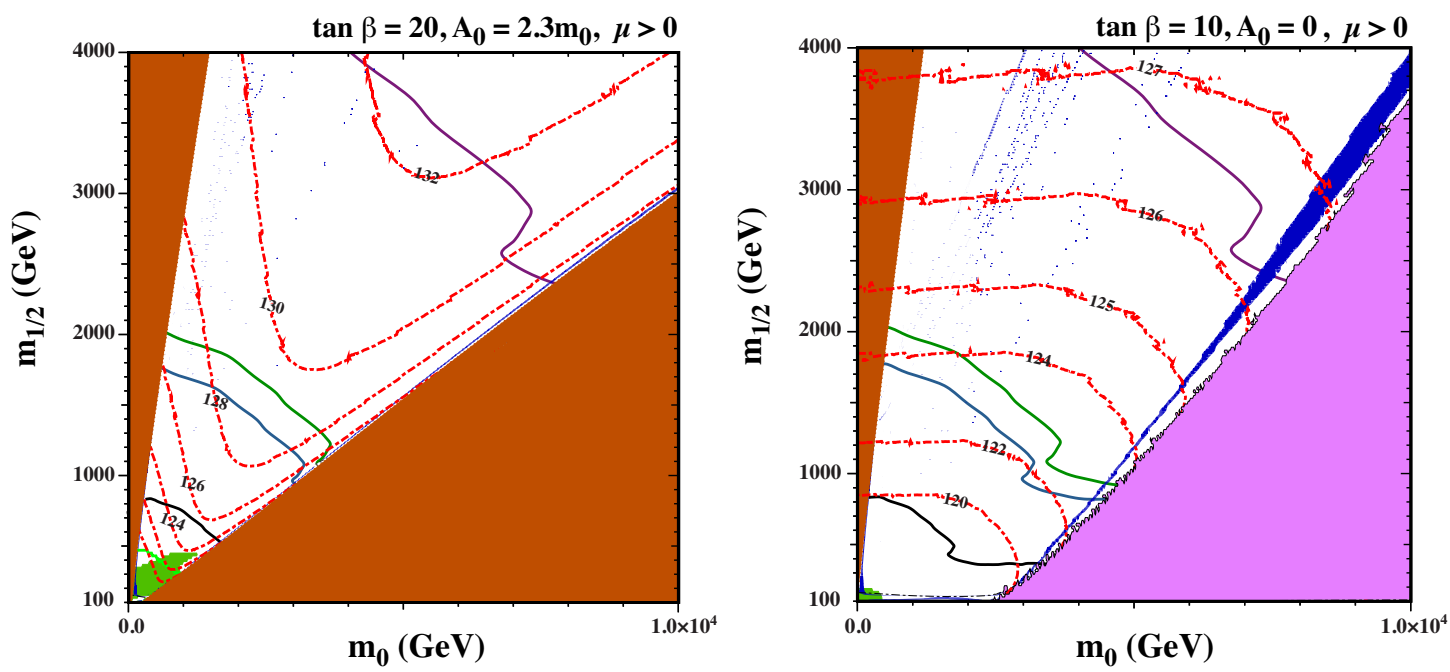

Figure 3: (left) The $\left(m_{0}, m_{1 / 2}\right)$ plane for fixed $\tan \beta=20$ and $A_{0}=2.3 m_{0}$. (right) The $\left(m_{0}, m_{1 / 2}\right)$ plane for fixed $\tan \beta=10$ and $A_{0}=0$. The dark red shaded regions are excluded because of a charged LSP and/or a tachyon, and the green region are excluded by $b \rightarrow s \gamma$ decay. There is no consistent electroweak vacuum in the purple region in the right panel. In the dark blue strips the relic LSP density lies within the range allowed by cosmology, and the dashed red lines are contours of $m_{h}$ as calculated using FeynHiggs 2.10 .0 [38]. The solid black, blue, green and purple lines in each panel are particle exclusion reaches for $\not_{T}$ searches with the LHC at $8 \mathrm{TeV}, 300$ and 3000/fb with LHC at $14 \mathrm{TeV}$, and 3000/fb with HE-LHC at $33 \mathrm{TeV}$, respectively.

The extent of the stau coannihilation strip is shown in the left panel of Fig. 4. There the stau-neutralino mass difference is plotted as a function of $m_{1 / 2}$ [37]. For this choice of $\tan \beta=10$, the strip extends only out to $m_{1 / 2} \simeq 900 \mathrm{GeV}$ and is all but excluded by the LHC (which excludes 
$m_{1 / 2} \lesssim 840 \mathrm{GeV}$ for relatively low $m_{0}$ [32]. At $\tan \beta=40$, the strip extends to slightly larger $m_{1 / 2}=1300 \mathrm{GeV}$ when $A_{0}=2.5 m_{0}$.
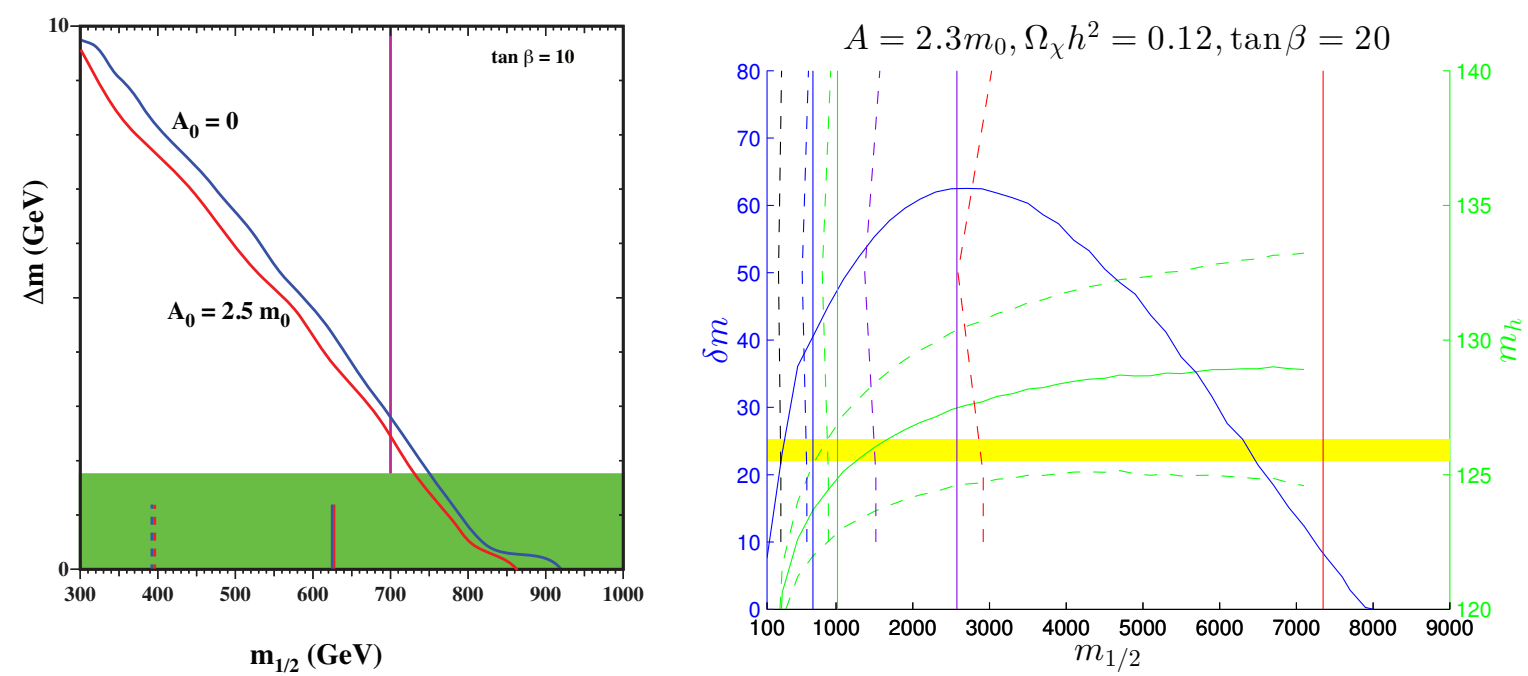

Figure 4: (left) The mass difference $\Delta m \equiv m_{\tilde{\tau}_{1}}-m_{\chi}$ as a function of $m_{1 / 2}$ along the CMSSM coannihilation strips for $\tan \beta=10$ and for $A_{0}=0$ (blue line) and $2.5 m_{0}$ (red line). The band with $m_{\tilde{\tau}_{1}}-m_{\chi}<m_{\tau}$ is shaded green. The lower limit on $m_{1 / 2}$ from the 8-TeV ATLAS $5 / \mathrm{fb} \not{ }_{T}$ search at the LHC [40] is represented by the maroon line, and the lower limits from searches for the direct and total production of metastable charged particles [41] are shown as dashed and solid lines, respectively, inside the green bands (see [37] for details). (right) The solid blue curve is the profile in the $\left(m_{1 / 2}, \delta m \equiv m_{\tilde{t}_{1}}-m_{\chi}\right)$ plane of the stop coannihilation strips for $A_{0} / m_{0}=2.3$ and $\tan \beta=20$. The near-vertical black, blue, green, purple and red lines in each panel are particle exclusion reaches for particle searches with LHC at $8 \mathrm{TeV}, 300$ and 3000/fb with LHC at $14 \mathrm{TeV}$, 3000/fb with HE-LHC at $33 \mathrm{TeV}$ and 3000/fb with FCC-hh at $100 \mathrm{TeV}$, respectively. The solid lines are for generic $\phi_{T}$ searches, and the dashed lines are for dedicated stop searches. The solid (dashed) near-horizontal green lines are central values (probable ranges) of $m_{h}$, and the yellow band represents the experimental value of $m_{h}[42]$.

When $A_{0}$ is large, one of the stop masses is driven small and the possibility for stop coannihilation is realized [43, 44, 39, 45]. The stop coannihilation strip is also seen in the $\left(m_{0}, m_{1 / 2}\right)$ plane in the left panel of Fig. 3. The stop strip corresponds to the thin blue line which tracks the dark red wedge in the lower right of the panel. This strip extends past the $m_{0}=10 \mathrm{TeV}$ extent of the figure. Unlike the stau strip, it is unlikely that the entire strip will be fully probed as it is seen to extend beyond the reach of a future $33 \mathrm{TeV}$ LHC upgrade. The full extent of the stop strip is seen in the right panel of Fig. 4 which shows the stop-neutralino mass difference as a function of $m_{1 / 2}$ [44, 39]. The stop strip extends to $\approx 8 \mathrm{TeV}$ in $m_{1 / 2}$ and $>20 \mathrm{TeV}$ in $m_{0}$.

Another difficulty for the stop strip seen in Fig. 5 is its detectability in direct detection experiments. The spin-independent cross section, $\sigma_{\mathrm{SI}}$, as a function of the neutralino mass is shown in the left panel of Fig. 5 for a similar case though here $\tan \beta=5$. The points in the panel represent results of a scan of the parameter space. Darker points fall within $3 \sigma$ of the dark matter relic density that fits best the Planck data [46]. Lighter points have smaller relic densities and should not be excluded. However, whenever the relic density is below the central value determined by Planck, we scale the cross section downwards by the ratio of the calculated density to the Planck density. 
The solid curve in Fig. 5 corresponds to the current LUX limit [27]. The thin black dashed curve corresponds to the projected LZ sensitivity $[28,30]$. The thick orange dashed line corresponds to the irreducible neutrino background [29, 30]. In this case, the LSP is almost pure bino and squarks are quite heavy and as a result the spin-independent cross section is quite small and generally falls below the neutrino background.
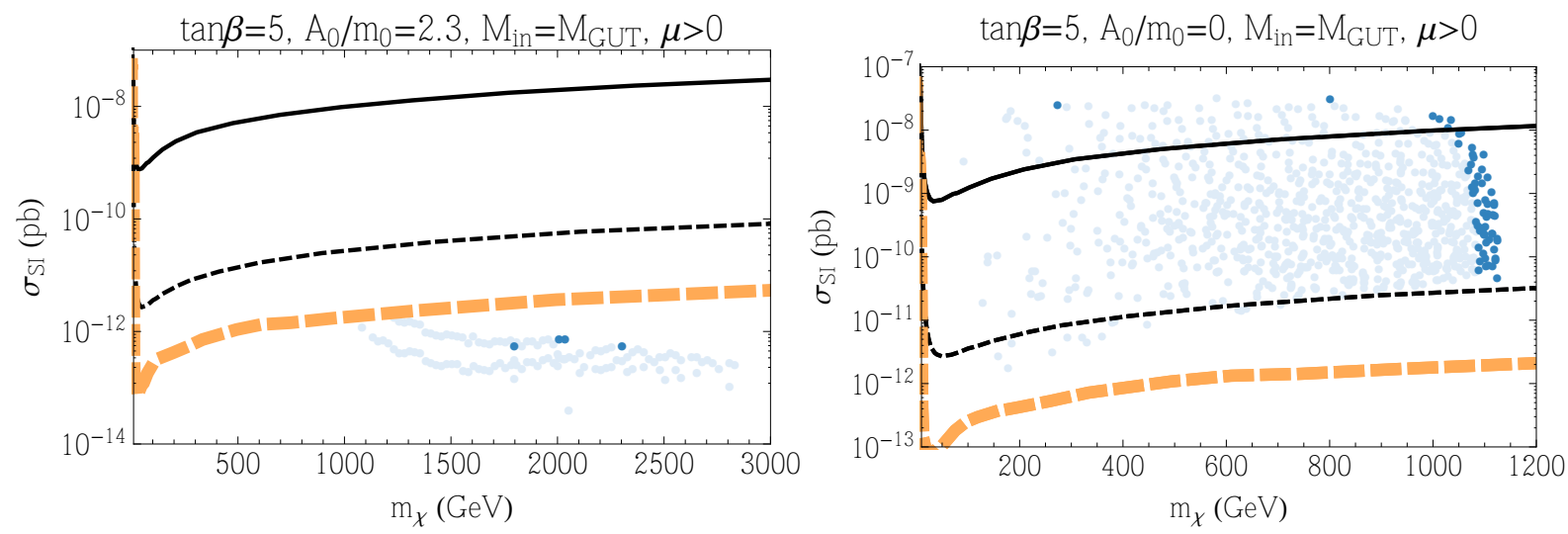

Figure 5: The spin-independent elastic scattering cross section in the CMSSM as a function of the neutralino mass for $\mu>0$, with $\tan \beta=5$ and $A_{0}=2.3 m_{0}$ (left) and $A_{0}=0$ (right). The panels show points where the relic density is within $3 \sigma$ of the central Planck value colored darker blue, and those where the relic density is below the Planck value as lighter blue points. The black solid curve is the current LUX bound. The black dashed curve is the projected LZ sensitivity and the dashed orange curve is the neutrino background level.

The focus point strip [6] occurs when $m_{0}$ is large and $A_{0}$ is relatively small. In this case, the minimization of the Higgs potential yields a relatively small value for $\mu$ so that the LSP becomes Higgsino-like. An example of the focus point strip is seen by the relatively thick blue strip in the right panel of Fig. 3. To the right of the strip, there is no solution to the Higgs minimization equations. In contrast to the stop coannihilation strip, the focus point strip is clearly within reach of direct detection experiments as seen in the right panel of Fig. 5. Almost all points sampled fall above the future reach of LZ [28, 30], though it should be noted that the constraint $m_{h}<128$ $\mathrm{GeV}$ was imposed allowing one to set a lower bound on the elastic cross section. The set of darkly shaded points with good relic density are found mostly at $m_{\chi} \simeq 1100 \mathrm{GeV}$ due to the fact that these points are mainly Higgsino LSPs [47].

In the CMSSM, with non-universal gaugino masses, it is possible that gluino coannihilations control the relic density $[48,45,49,50]$. For example, by allowing the input gluino mass to differ from the bino and wino masses at the GUT scale $\left(M_{1}=M_{2} \neq M_{3}\right)$, the gluino may be the next lightest superpartner and opens the possibility for gluino coannihilations. In the left panel of Fig. 6 , we show an example of a $\left(M_{1}, M_{3}\right)$ plane for fixed $\tan \beta=3, A_{0} / m_{0}=1.5$, and $m_{0}=200 \mathrm{TeV}$ [50]. The gluino-bino coannihilation strip is seen as the thin blue strip following the gluino LSP region shown in dark red and extends up to $\sim 3 \mathrm{TeV}$. The panel on the right shows the gluinoneutralino mass difference $\Delta M$ (blue line) which peaks at approximately $170 \mathrm{GeV}$, and is consistent with the results of [49] for intermediate squark-to-gluino mass ratios. Also shown is the neutralino mass as a function of $M_{3}$ (red line): it rises to $m_{\chi} \sim 8 \mathrm{TeV}$ at the tip of the coannihilation strip. The Higgs mass (which is not shown) varies very slowly across the $\left(M_{1}, M_{3}\right)$ plane and takes the 
value $m_{h}=125$ for this value of $\tan \beta$. At lower values of $m_{0}$, more possibilities arise, namely, at large $M_{1}$, the composition of the LSP changes from bino to Higgsino, and the possibility of gluinoHiggsino coannihilations appear. At still larger $M_{1}$ (for fixed $M_{3}$, there is a also a focus point region [50].
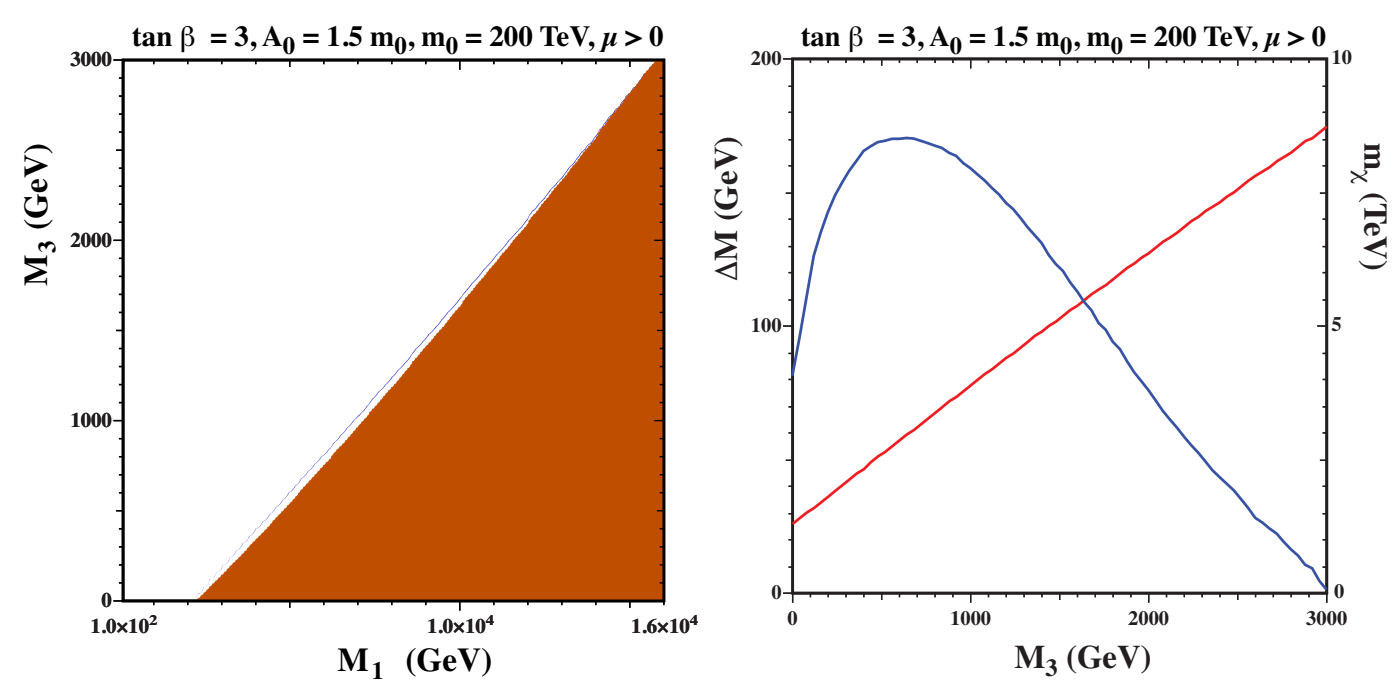

Figure 6: The $\left(M_{1}, M_{3}\right)$ plane (left) for $m_{0}=200 \mathrm{TeV}, A_{0} / m_{0}=1.5$ and $\tan \beta=3$. The dark blue strip in the left panel shows where the relic LSP density $\Omega_{\chi} h^{2}$ falls within the $\pm 3-\sigma$ range allowed by Planck and other data, and the lightest neutralino is no longer the LSP in the regions shaded brick-red. The right panel shows the gluino-neutralino mass difference (left axis, blue line) and the neutralino mass (right axis, red line) as functions of $M_{3}$.

At large $\tan \beta$, it is also common that there are regions of the parameter space where the LSP mass is very close to $m_{A} / 2$, where $m_{A}$ is the Higgs pseudoscalar mass. In this case, there is a large contribution to the cross section from rapid s-channel annihilations, producing two strips (known as the funnel region) on either side of the pole [1]. However, at large $\tan \beta$, there are strong constraints from $B_{s} \rightarrow \mu^{+} \mu^{-}$decay [51]. This region will not be discussed further here.

There are of course many other possibilities in CMSSM-like models. These include models where the Higgs mass are non-universal: the NUHM1 $\left(m_{1}=m_{2} \neq m_{0}\right)[52,53,3,4]$, and NUHM2 $\left(m_{1} \neq m_{2} \neq m_{0}\right)[54,53,3,4]$; subGUT models where the input universality scale differs from the GUT scale with $M_{\text {in }}<M_{G U T}$ [55, 3, 4]; superGUT models with $M_{\text {in }}>M_{G U T}$ [56]. The above mentioned models all have 1-2 additional parameters relative to the CMSSM. However there are also viable models with fewer than the 4 free CMSSM parameters: These include mSUGRA models, where the condition that $B_{0}=A_{0}-m_{0}$ is applied and hence $\tan \beta$ is no longer free $[57,58,3,4]$; and pure gravity mediated models [59] for which gaugino masses are generated through anomalies [60] on top of the mSUGRA conditions [61] and produces a spectrum reminiscent of split supersymmetry [62].

\section{SO(10) Dark Matter}

The motivations for supersymmetry are well known. These include the possibility for gauge coupling unification at the GUT scale [63], the stability of the electroweak vacuum [64], radiative 
electroweak symmetry breaking [65]; a stable dark matter candidate [66], the stabilization of the gauge hierarchy [67]. With the exception of the latter, non-supersymmetric SO(10) GUT [7] models may contain equivalences of all of these desirable features. In models with an intermediate scale between the electroweak scale and the GUT scale, gauge coupling unification becomes possible [9] when the intermediate scale is determined by the unification conditions given a field content below the GUT scale. As discussed in more detail below, in $\mathrm{SO}(10)$ models where the intermediate scale is broken by a Higgs in a $\mathbf{1 2 6}$ representation, a residual $Z_{2}$ discrete symmetry survives enabling the possibility of a stable dark matter candidate [16, 10, 11, 12]. Furthermore, in models with gauge coupling unification and a stable dark matter candidate, it is also possible to stabilize the electroweak vacuum while at the same time radiatively break the electroweak symmetry [13]. In addition, one has the additional benefit of the seesaw mechanism for generating neutrino masses [14].

To construct an $\mathrm{SO}(10)$ dark matter model, we should first pick an intermediate scale gauge group and a representation for the Higgs field, $R_{1}$ which breaks $\mathrm{SO}(10)$. The possibilities considered are listed in Table 1.

Table 1: Candidates for the intermediate gauge group $G_{\text {int }}$.

\begin{tabular}{ll}
\hline \hline$G_{\text {int }}$ & $R_{1}$ \\
\hline$S U(4)_{C} \otimes S U(2)_{L} \otimes S U(2)_{R}$ & $\mathbf{2 1 0}$ \\
$S U(4)_{C} \otimes S U(2)_{L} \otimes S U(2)_{R} \otimes D$ & $\mathbf{5 4}$ \\
$S U(4)_{C} \otimes S U(2)_{L} \otimes U(1)_{R}$ & $\mathbf{4 5}$ \\
$S U(3)_{C} \otimes S U(2)_{L} \otimes S U(2)_{R} \otimes U(1)_{B-L}$ & $\mathbf{4 5}$ \\
$S U(3)_{C} \otimes S U(2)_{L} \otimes S U(2)_{R} \otimes U(1)_{B-L} \otimes D$ & $\mathbf{2 1 0}$ \\
$S U(3)_{C} \otimes S U(2)_{L} \otimes U(1)_{R} \otimes U(1)_{B-L}$ & $\mathbf{4 5 , 2 1 0}$ \\
\hline$S U(5) \otimes U(1)$ & $\mathbf{4 5 , 2 1 0}$ \\
Flipped $S U(5) \otimes U(1)$ & $\mathbf{4 5 , 2 1 0}$ \\
\hline \hline
\end{tabular}

As noted above, we must employ a $\mathbf{1 2 6}$ to break the intermediate gauge group down to the SM in order to preserve a $Z_{2}$ symmetry related to matter parity. The coupling of the $\mathbf{1 2 6}$ to SM matter fields embedded in a $\mathbf{1 6}$ representation of $\mathrm{SO}(10)$ naturally gives rise to a majorana mass mass to the $v_{R}$ component of the $\mathbf{1 6}$ of order $\langle\mathbf{1 2 6}\rangle \sim M_{\text {int }}$ which when combined with the Dirac mass arising from the vev of the SM Higgs (now residing in a 10-plet of $\mathrm{SO}(10)$ ) gives rise to the seesaw mechanism for light neutrino masses [14].

Next we must choose a representation for the dark matter candidate. Possible choices are given in Table 2. A fermionic DM candidate should be parity even and belong to a 10, 45, 54, 120, 126, 210 or $\mathbf{2 1 0}$ ' representation, while scalar DM is parity odd and belongs to a $\mathbf{1 6}$ or $\mathbf{1 4 4}$ representation. Following the branching rules given in Ref. [68], in Table 2, we list $S U(2)_{L} \otimes U(1)_{Y}$ multiplets in various $\mathrm{SO}(10)$ representations that contain an electrically neutral color singlet. The table is classified by $B-L$ so one can check the matter parity of the candidates easily; $B-L=$ 0,2 candidates are fermionic while $B-L=1$ candidates are scalar, labeled by an " $F$ " or " $S$ " at the beginning of each row, respectively. The subscript of the model names denotes the $S U(2)_{L}$ representation, while the superscript shows hypercharge. A hat is used for $B-L=2$ candidates. 
Table 2: List of $S U(2)_{L} \otimes U(1)_{Y}$ multiplets in $S O(10)$ representations that contain an electric neutral color singlet.

\begin{tabular}{lccll}
\hline \hline Model & $B-L$ & $S U(2)_{L}$ & $Y$ & $\mathrm{SO}(10)$ representations \\
\hline $\mathrm{F}_{\mathbf{1}}^{0}$ & & $\mathbf{1}$ & 0 & $\mathbf{4 5}, \mathbf{5 4 , \mathbf { 2 1 0 }}$ \\
$\mathrm{F}_{\mathbf{2}}^{1 / 2}$ & & $\mathbf{2}$ & $1 / 2$ & $\mathbf{1 0}, \mathbf{1 2 0}, \mathbf{1 2 6}, \mathbf{2 1 0}^{\prime}$ \\
$\mathrm{F}_{\mathbf{3}}^{0}$ & 0 & $\mathbf{3}$ & 0 & $\mathbf{4 5}, \mathbf{5 4}, \mathbf{2 1 0}$ \\
$\mathrm{F}_{\mathbf{3}}^{1}$ & & $\mathbf{3}$ & 1 & $\mathbf{5 4}$ \\
$\mathrm{F}_{\mathbf{4}}^{1 / 2}$ & & $\mathbf{4}$ & $1 / 2$ & $\mathbf{2 1 0}^{\prime}$ \\
$\mathrm{F}_{\mathbf{4}}^{3 / 2}$ & & $\mathbf{4}$ & $3 / 2$ & $\mathbf{2 1 0}$ \\
\hline $\mathrm{S}_{\mathbf{1}}^{0}$ & & $\mathbf{1}$ & 0 & $\mathbf{1 6}, \mathbf{1 4 4}$ \\
$\mathrm{S}_{\mathbf{2}}^{1 / 2}$ & 1 & $\mathbf{2}$ & $1 / 2$ & $\mathbf{1 6}, \mathbf{1 4 4}$ \\
$\mathrm{S}_{\mathbf{3}}^{0}$ & & $\mathbf{3}$ & 0 & $\mathbf{1 4 4}$ \\
$\mathrm{S}_{\mathbf{3}}^{1}$ & & $\mathbf{3}$ & 1 & $\mathbf{1 4 4}$ \\
\hline$\widehat{\mathrm{F}}_{\mathbf{1}}^{0}$ & & $\mathbf{1}$ & 0 & $\mathbf{1 2 6}$ \\
$\widehat{\mathrm{F}}_{\mathbf{2}}^{1 / 2}$ & 2 & $\mathbf{2}$ & $1 / 2$ & $\mathbf{2 1 0}$ \\
$\widehat{\mathrm{F}}_{\mathbf{3}}^{1}$ & & $\mathbf{3}$ & 1 & $\mathbf{1 2 6}$ \\
\hline \hline
\end{tabular}

Depending on the dark matter and Higgs representation chosen, renormalization group evolution of the gauge couplings can be used to determine, the GUT scale, the intermediate scale, and the value of the GUT gauge coupling. One such example is a singlet fermion $\left(\mathrm{F}_{\mathbf{1}}^{0}\right)$ originating in the $(\mathbf{1 5}, \mathbf{1}, \mathbf{1})$ representation (in terms of $S U(4) \otimes S U(2) \otimes S U(2)$ ) included in the $\mathbf{4 5}$ of $S O(10)$. The evolution of the gauge couplings in this model is shown in Figure 7. In this model [11], $R_{1}=$ 54, and we have $\log \left(M_{\text {int }}\right)=13.66, \log \left(M_{G U T}\right)=15.87$, and $g_{G U T}=0.567$. For further details concerning this model, see [11].

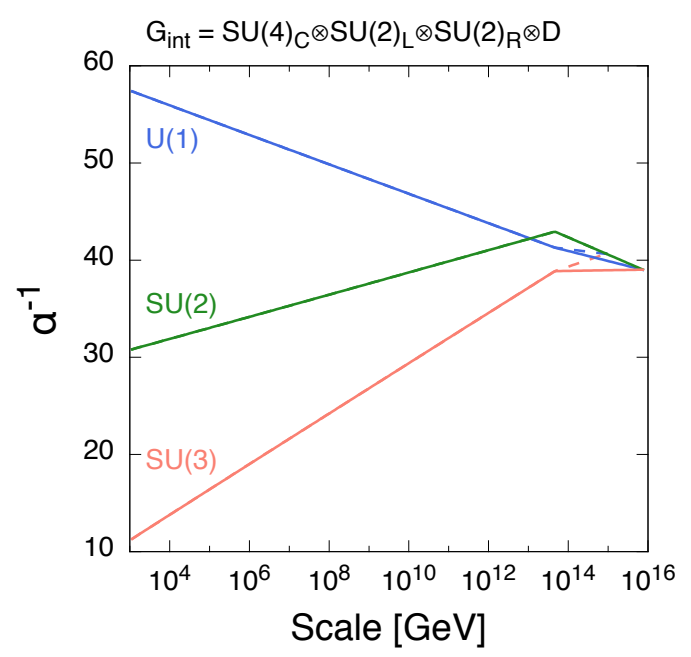

Figure 7: Running of the gauge couplings for the fermionic singlet dark matter model described in the text. 
Given the large number of possible intermediate gauge groups and the large number of possible dark matter representations (scalar or fermionic), one may think that there are a vast number of dark matter models in the $\mathrm{SO}(10)$ framework. However, once we demand gauge coupling unification, there are in fact only a handful of models which permit gauge coupling unification and satisfy constraints from the lifetime of the proton. We also require that the solution give $M_{\text {int }}<M_{G U T}$ and that we can split the SO(10) multiplets in such a way to leave only the DM candidate (and perhaps weak partners) with weak scale masses. The resulting acceptable models for scalar dark matter candidates is shown in Table 3. For more information on these models see [12]. The singlet models (SA) have a phenomenology similar to that of so-called Higgs portal models [71]. To roughly estimate the favored mass region for a scalar singlet, consider the quartic interaction between the singlet DM $\phi$ and the SM Higgs field: $\lambda_{H \phi} \phi^{2}|H|^{2} / 2$. Through this coupling, the singlet DM particles annihilate into a pair of the SM Higgs bosons and weak gauge bosons. The annihilation cross section is

$$
\sigma_{\text {ann }} v_{\text {rel }} \simeq \frac{\lambda_{H \phi}^{2}}{16 \pi m_{D M}^{2}},
$$

assuming that the DM mass $m_{D M}$ is much larger than the SM Higgs mass $m_{h}$ and neglecting terms proportional to $v^{2}$. The DM relic abundance is, on the other hand, related to the annihilation cross section by

$$
\Omega_{D M} h^{2} \simeq \frac{3 \times 10^{-27} \mathrm{~cm}^{3} \mathrm{~s}^{-1}}{\left\langle\sigma_{a n n} v_{r e l}\right\rangle} .
$$

To account for the observed DM density $\Omega_{D M} h^{2}=0.12$ [46], the DM mass should be $m_{D M} \lesssim$ $10 \mathrm{TeV}$ for $\lambda_{H \phi} \lesssim 1$. This gives us a rough upper bound for the DM mass. The other scalar DM candidates are $S U(2)_{L} \otimes U(1)_{Y}$ multiplets, which can interact with SM particles through gauge interactions in addition to the quartic coupling mentioned above. In particular, $\mathrm{S}_{2}^{1 / 2}$ is known as the Inert Higgs Doublet Model and has been widely studied in the literature [72, 73].

Table 3: One-loop result for $M_{G U T}, M_{\text {int }}, \alpha_{G U T}$, and proton lifetimes for scalar dark matter models. The $D M$ mass is set to be $m_{D M}=1 \mathrm{TeV}$. The mass scales are given in $\mathrm{GeV}$ and the proton lifetimes are in units of years. These models evade the proton decay bound, $\tau\left(p \rightarrow e^{+} \pi^{0}\right)>1.4 \times 10^{34}$ yrs[69, 70].

\begin{tabular}{lcllll}
\hline \hline Model & $R_{\mathrm{DM}}$ & $\log _{10} M_{\mathrm{GUT}}$ & $\log _{10} M_{\text {int }}$ & $\alpha_{\mathrm{GUT}}$ & $\log _{10} \tau_{p}\left(p \rightarrow e^{+} \pi^{0}\right)$ \\
\hline \hline \multicolumn{5}{c}{$G_{\text {int }}=S U(4)_{C} \otimes S U(2)_{L} \otimes S U(2)_{R}$} \\
\hline $\mathrm{SA}_{422}\left(\mathrm{~S}_{\mathbf{1}}^{0}\right)$ & $\mathbf{4 , 1 , 2}$ & 16.33 & 11.08 & 0.0218 & $36.8 \pm 1.2$ \\
$\mathrm{SB}_{422}\left(\mathrm{~S}_{\mathbf{2}}^{1 / 2}\right)$ & $\mathbf{4 , 2}, \mathbf{1}$ & 15.62 & 12.38 & 0.0228 & $34.0 \pm 1.2$ \\
\hline \multicolumn{5}{c}{$G_{\text {int }}=S U(3)_{C} \otimes S U(2)_{L} \otimes S U(2)_{R} \otimes U(1)_{B-L}$} \\
\hline $\mathrm{SA}_{3221}\left(\mathrm{~S}_{\mathbf{1}}^{0}\right)$ & $\mathbf{1 , 1 , 2}, 1$ & 16.66 & 8.54 & 0.0217 & $38.1 \pm 1.2$ \\
$\mathrm{SB}_{3221}\left(\mathrm{~S}_{\mathbf{2}}^{1 / 2}\right)$ & $\mathbf{1}, \mathbf{2}, \mathbf{1},-1$ & 16.17 & 9.80 & 0.0223 & $36.2 \pm 1.2$ \\
$\mathrm{SC}_{3221}\left(\mathrm{~S}_{\mathbf{2}}^{1 / 2}\right)$ & $\mathbf{1}, \mathbf{2}, \mathbf{3},-1$ & 15.62 & 9.14 & 0.0230 & $34.0 \pm 1.2$ \\
\hline \multicolumn{5}{c}{$G_{\text {int }}=S U(3)_{C} \otimes S U(2)_{L} \otimes S U(2)_{R} \otimes U(1)_{B-L} \otimes D$} \\
\hline $\mathrm{SA}_{3221 \mathrm{D}}\left(\mathrm{S}_{\mathbf{1}}^{0}\right)$ & $\mathbf{1 , 1 , 2}, 1$ & 15.58 & 10.08 & 0.0231 & $33.8 \pm 1.2$ \\
$\mathrm{SB}_{3221 \mathrm{D}}\left(\mathrm{S}_{2}^{1 / 2}\right)$ & $\mathbf{1 , 2}, \mathbf{1},-1$ & 15.40 & 10.44 & 0.0233 & $33.1 \pm 1.2$ \\
\hline \hline
\end{tabular}

An example of a fermionic singlet was discussed above, and its relic density is mainly determined by the reheat temperature after inflation (see [10, 11] for more details). Non-singlet fermions 
behaving as well studied wimps are also possible. From Table 2, we see that the only candidates without hypercharge is the weak (wino-like) triplet $\mathrm{F}_{3}^{0}$. One example of a triplet candidate is given in Table 4. As one can see, this state is a singlet under both $\mathrm{SU}(4)_{C}$ and $\mathrm{SU}(2)_{R}$ and originates in a 45 of $\mathrm{SO}(10)$. While the intermediate scale is relatively low, the GUT scale is quite high and hence the proton lifetime is unobservably long.

Table 4: The one-loop results for $M_{G U T}, M_{i n t}, \alpha_{G U T}$, and proton lifetimes for a real triplet fermionic DM models. Here the DM mass is set to be $1 \mathrm{TeV}$. The mass scales and proton decay lifetime are in units of GeV and years, respectively.

\begin{tabular}{lccccc}
\hline \hline Model & $R_{\mathrm{DM}}$ & $\log _{10} M_{\text {int }}$ & $\log _{10} M_{\mathrm{GUT}}$ & $\alpha_{\mathrm{GUT}}$ & $\log _{10} \tau_{p}\left(p \rightarrow e^{+} \pi^{0}\right)$ \\
\hline \hline \multicolumn{5}{c}{$G_{\text {int }}=\mathrm{SU}(4)_{C} \otimes \mathrm{SU}(2)_{L} \otimes \mathrm{SU}(2)_{R}$} \\
\hline $\mathrm{F}_{\mathbf{3}}^{0}$ & $(\mathbf{1}, \mathbf{3}, \mathbf{1})$ & 6.54 & 17.17 & 0.0252 & $39.8 \pm 1.2$ \\
\hline \hline
\end{tabular}

It is also possible that a fermionic dark matter candidate carries hypercharge and in this case, it may be either a weak doublet (Higgsino-like) or triplet. Some examples are shown in Table 5. In this case, we must introduce another representation (at the intermediate scale) to mix with $R_{\mathrm{DM}}$ in order to induce some splitting in the DM multiplet to evade current DM detection experimental results. These are denoted $R_{\mathrm{DM}}^{\prime}$ in the table.

Table 5: Possible hypercharged fermionic DM models that are not yet excluded by current proton decay experiments. The quantum numbers are labeled in the same order as $G_{\text {int }}$. The subscripts $D$ and $W$ refer to Dirac and Weyl respectively. The numerical results are calculated for DM mass of $1 \mathrm{TeV}$. The mass scales and proton decay lifetime are in unit of GeV and years, respectively.

\begin{tabular}{ccccccc}
\hline \hline Model & $R_{\mathrm{DM}}$ & $R_{\mathrm{DM}}^{\prime}$ & $\log _{10} M_{\text {int }}$ & $\log _{10} M_{\mathrm{GUT}}$ & $\alpha_{\mathrm{GUT}}$ & $\log _{10} \tau_{p}$ \\
\hline \hline $\mathrm{FA}_{421}\left(\mathrm{~F}_{\mathbf{2}}^{1 / 2}\right)$ & $(\mathbf{1}, \mathbf{2}, 1 / 2)_{D}$ & $(\mathbf{1 5}, \mathbf{1}, 0)_{W}$ & 3.48 & 17.54 & 0.0320 & $40.9 \pm 1.2$ \\
\hline \multicolumn{7}{c}{$G_{\text {int }}=\mathrm{SU}(4)_{C} \otimes \mathrm{SU}(2)_{L} \otimes \mathrm{SU}(2)_{R}$} \\
\hline $\mathrm{FA}_{422}\left(\mathrm{~F}_{\mathbf{2}}^{1 / 2}\right)$ & $(\mathbf{1}, \mathbf{2}, \mathbf{2})_{W}$ & $(\mathbf{1}, \mathbf{3}, \mathbf{1})_{W}$ & 9.00 & 15.68 & 0.0258 & $34.0 \pm 1.2$ \\
\hline $\mathrm{FB}_{422}\left(\mathrm{~F}_{\mathbf{2}}^{1 / 2}\right)$ & $(\mathbf{1}, \mathbf{2}, \mathbf{2})_{W}$ & $(\mathbf{1}, \mathbf{3}, \mathbf{1})_{W}$ & 5.84 & 17.01 & 0.0587 & $38.0 \pm 1.2$ \\
\hline \hline
\end{tabular}

\section{Summary}

It is becoming apparent that recent LHC searches for supersymmetry have pushed CMSSM into corners of the parameter space which rely on the near degeneracy between the LSP and the next lightest superpartner, thus allowing coannihilations to reign in the relic density. While the stau coannihilation strip is nearly ruled out by LHC searches, possibilities remain for the stop strip and if there are non-universal gaugino masses, gluino coannihilation. It is also possible that $m_{0}$ is large near the focus point strip so that the LSP is mostly Higgsino-like. Though not discussed here, there are several variants of the CMSSM which still permit neutralino dark matter. These include models with non-universal Higgs scalar masses (NUHM), models where the input universality scale is below the GUT scale (subGUT models), or pure gravity mediated models with either wino or Higgsino dark matter. 
While supersymmetry has many motivations beyond dark matter, with the exception of the hierarchy problem, almost of these motivating factors can be resolved in non-supersymmetric version of $\mathrm{SO}(10)$ grand unifications. Several such examples were outlined above. The real challenge lies in the detection of dark matter and our ability to discriminate between the various models.

\section{References}

[1] M. Drees and M. M. Nojiri, Phys. Rev. D 47 (1993) 376 [arXiv:hep-ph/9207234]; H. Baer and M. Brhlik, Phys. Rev. D 53 (1996) 597 [arXiv:hep-ph/9508321]; Phys. Rev. D 57 (1998) 567 [arXiv:hep-ph/9706509]; H. Baer, M. Brhlik, M. A. Diaz, J. Ferrandis, P. Mercadante, P. Quintana and X. Tata, Phys. Rev. D 63 (2001) 015007 [arXiv:hep-ph/0005027]; J. R. Ellis, T. Falk, G. Ganis, K. A. Olive and M. Srednicki, Phys. Lett. B 510 (2001) 236 [arXiv:hep-ph/0102098].

[2] G. L. Kane, C. F. Kolda, L. Roszkowski and J. D. Wells, Phys. Rev. D 49 (1994) 6173 [arXiv:hep-ph/9312272]; J. R. Ellis, T. Falk, K. A. Olive and M. Schmitt, Phys. Lett. B 388 (1996) 97 [arXiv:hep-ph/9607292]; Phys. Lett. B 413 (1997) 355 [arXiv:hep-ph/9705444]; V. D. Barger and C. Kao, Phys. Rev. D 57 (1998) 3131 [arXiv:hep-ph/9704403]; L. Roszkowski, R. Ruiz de Austri and T. Nihei, JHEP 0108 (2001) 024 [arXiv:hep-ph/0106334]; A. Djouadi, M. Drees and J. L. Kneur, JHEP 0108 (2001) 055 [arXiv:hep-ph/0107316]; U. Chattopadhyay, A. Corsetti and P. Nath, Phys. Rev. D 66 (2002) 035003 [arXiv:hep-ph/0201001]; J. R. Ellis, K. A. Olive and Y. Santoso, New Jour. Phys. 4 (2002) 32 [arXiv:hep-ph/0202110]; H. Baer, C. Balazs, A. Belyaev, J. K. Mizukoshi, X. Tata and Y. Wang, JHEP 0207 (2002) 050 [arXiv:hep-ph/0205325]; R. Arnowitt and B. Dutta, arXiv:hep-ph/0211417; J. R. Ellis, T. Falk, G. Ganis, K. A. Olive and M. Schmitt, Phys. Rev. D 58 (1998) 095002 [arXiv:hep-ph/9801445]; J. R. Ellis, T. Falk, G. Ganis and K. A. Olive, Phys. Rev. D 62 (2000) 075010 [arXiv:hep-ph/0004169]; J. R. Ellis, K. A. Olive, Y. Santoso and V. C. Spanos, Phys. Lett. B 565 (2003) 176 [arXiv:hep-ph/0303043]; H. Baer and C. Balazs, JCAP 0305, 006 (2003) [arXiv:hep-ph/0303114]; A. B. Lahanas and D. V. Nanopoulos, Phys. Lett. B 568, 55 (2003) [arXiv:hep-ph/0303130]; U. Chattopadhyay, A. Corsetti and P. Nath, Phys. Rev. D 68, 035005 (2003) [arXiv:hep-ph/0303201]; C. Munoz, Int. J. Mod. Phys. A 19, 3093 (2004) [arXiv:hep-ph/0309346]; R. Arnowitt, B. Dutta and B. Hu, arXiv:hep-ph/0310103; J. Ellis and K. A. Olive, arXiv:1001.3651 [astro-ph.CO], published in Particle dark matter, ed. G. Bertone, pp. 142-163; J. Ellis and K. A. Olive, Eur. Phys. J. C 72, 2005 (2012) [arXiv:1202.3262 [hep-ph]]; O. Buchmueller et al., Eur. Phys. J. C 74 (2014) 3, 2809 [arXiv:1312.5233 [hep-ph]].

[3] J. Ellis, F. Luo, K. A. Olive and P. Sandick, Eur. Phys. J. C 73, no. 4, 2403 (2013) [arXiv:1212.4476 [hep-ph]].

[4] J. Ellis, J. L. Evans, F. Luo, N. Nagata, K. A. Olive and P. Sandick, Eur. Phys. J. C 76, no. 1, 8 (2016) doi:10.1140/epjc/s10052-015-3842-6 [arXiv:1509.08838 [hep-ph]].

[5] K. Griest and D. Seckel, Phys. Rev. D 43, 3191 (1991).

[6] J. L. Feng, K. T. Matchev and T. Moroi, Phys. Rev. Lett. 84, 2322 (2000) [arXiv:hep-ph/9908309]; Phys. Rev. D 61, 075005 (2000) [arXiv:hep-ph/9909334]; J. L. Feng, K. T. Matchev and F. Wilczek, Phys. Lett. B 482, 388 (2000) [arXiv:hep-ph/0004043]; H. Baer, T. Krupovnickas, S. Profumo and P. Ullio, JHEP 0510 (2005) 020 [hep-ph/0507282]; J. L. Feng, K. T. Matchev and D. Sanford, Phys. Rev. D 85, 075007 (2012) [arXiv:1112.3021 [hep-ph]]; P. Draper, J. Feng, P. Kant, S. Profumo and D. Sanford, Phys. Rev. D 88, 015025 (2013) [arXiv:1304.1159 [hep-ph]].

[7] H. Georgi, AIP Conf. Proc. 23, 575 (1975); H. Fritzsch and P. Minkowski, Annals Phys. 93, 193 (1975); M. S. Chanowitz, J. R. Ellis and M. K. Gaillard, Nucl. Phys. B 128, 506 (1977); H. Georgi and D. V. Nanopoulos, Nucl. Phys. B 155, 52 (1979). 
[8] H. Georgi and D. V. Nanopoulos, Nucl. Phys. B 159, 16 (1979); C. E. Vayonakis, Phys. Lett. B 82, 224 (1979) [Phys. Lett. 83B, 421 (1979)].

[9] A. Masiero, Phys. Lett. B 93, 295 (1980); Q. Shafi, M. Sondermann and C. Wetterich, Phys. Lett. B 92, 304 (1980); F. del Aguila and L. E. Ibanez, Nucl. Phys. B 177, 60 (1981); R. N. Mohapatra and G. Senjanovic, Phys. Rev. D 27, 1601 (1983); M. Fukugita and T. Yanagida, In *Fukugita, M. (ed.), Suzuki, A. (ed.): Physics and astrophysics of neutrinos* 1-248. and Kyoto Univ. - YITP-K-1050 (93/12,rec.Feb.94) 248 p. C.

[10] Y. Mambrini, K. A. Olive, J. Quevillon and B. Zaldivar, Phys. Rev. Lett. 110 (2013) 24, 241306 [arXiv:1302.4438 [hep-ph]].

[11] Y. Mambrini, N. Nagata, K. A. Olive, J. Quevillon and J. Zheng, Phys. Rev. D 91, no. 9, 095010 (2015) [arXiv:1502.06929 [hep-ph]].

[12] N. Nagata, K. A. Olive and J. Zheng, JHEP 1510, 193 (2015) [arXiv:1509.00809 [hep-ph]].

[13] Y. Mambrini, N. Nagata, K. A. Olive and J. Zheng, arXiv:1602.05583 [hep-ph].

[14] P. Minkowski, Phys. Lett. B 67 (1977) 421; M. Gell-Mann, P. Ramond and R. Slansky, in Supergravity, eds. D. Freedman and P. Van Nieuwenhuizen (North Holland, Amsterdam, 1979), pp. 315-321. ISBN 044485438x; T. Yanagida, in Proceedings of the Workshop on the Unified Theory and The Baryon Number of the Universe, eds O. Sawada and S. Sugamoto. KEK79-18 (1979); R. N. Mohapatra and G. Senjanovic, Phys. Rev. Lett. 44, 912 (1980); J. Schechter and J. W. F. Valle, Phys. Rev. D 22 (1980) 2227; J. Schechter and J. W. F. Valle, Phys. Rev. D 25 (1982) 774.

[15] T. W. B. Kibble, G. Lazarides and Q. Shafi, Phys. Lett. B 113, 237 (1982). L. M. Krauss and F. Wilczek, Phys. Rev. Lett. 62, 1221 (1989); L. E. Ibanez and G. G. Ross, Phys. Lett. B 260, 291 (1991); L. E. Ibanez and G. G. Ross, Nucl. Phys. B 368, 3 (1992); S. P. Martin, Phys. Rev. D 46, 2769 (1992) [hep-ph/9207218]; M. De Montigny and M. Masip, Phys. Rev. D 49, 3734 (1994) [hep-ph/9309312].

[16] M. Kadastik, K. Kannike and M. Raidal, Phys. Rev. D 80 (2009) 085020 [Erratum-ibid. D 81 (2010) 029903] [arXiv:0907.1894 [hep-ph]]; M. Kadastik, K. Kannike and M. Raidal, Phys. Rev. D 81, 015002 (2010) [arXiv:0903.2475 [hep-ph]]; M. Frigerio and T. Hambye, Phys. Rev. D 81 (2010) 075002 [arXiv:0912.1545 [hep-ph]].

[17] J. L. Evans, N. Nagata, K. A. Olive and J. Zheng, JHEP 1602, 120 (2016) doi:10.1007/JHEP02(2016)120 [arXiv:1512.02184 [hep-ph]].

[18] O. Buchmueller et al., Eur. Phys. J. C 64, 391 (2009) [arXiv:0907.5568 [hep-ph]].

[19] O. Buchmueller et al., Eur. Phys. J. C 74 (2014) 12, 3212 [arXiv:1408.4060 [hep-ph]]; E. A. Bagnaschi et al., Eur. Phys. J. C 75, 500 (2015) doi:10.1140/epjc/s10052-015-3718-9 [arXiv:1508.01173 [hep-ph]].

[20] For more information and updates, please see http://cern. ch/mastercode/.

[21] John R. Ellis, Giovanni Ridolfi and Fabio Zwirner, Phys. Lett. B257, 83, 1991; John R. Ellis, Giovanni Ridolfi and Fabio Zwirner, Phys. Lett. B262, 477, 1991; Y. Okada, Masahiro Yamaguchi and T. Yanagida, Phys. Lett. B262, 54, 1991; Yasuhiro Okada, Masahiro Yamaguchi and Tsutomu Yanagida, Prog. Theor. Phys. 85, 1, 1991; Howard E. Haber and Ralf Hempfling, Phys. Rev. Lett. 66, $1815,1991$.

[22] J. R. Ellis, D. V. Nanopoulos, K. A. Olive and Y. Santoso, Phys. Lett. B 633, 583 (2006) [arXiv:hep-ph/0509331]. 
[23] Z. Ahmed et al. [CDMS Collaboration], Phys. Rev. Lett. 102, 011301 (2009) [arXiv:0802.3530 [astro-ph]].

[24] J. Angle et al. [XENON Collaboration], Phys. Rev. Lett. 100, 021303 (2008) [arXiv:0706.0039 [astro-ph]].

[25] E. Aprile et al. [XENON100 Collaboration], Phys. Rev. Lett. 107, 131302 (2011) [arXiv:1104.2549 [astro-ph.CO]].

[26] E. Aprile et al. [XENON100 Collaboration], Phys. Rev. Lett. 109, 181301 (2012) [arXiv:1207.5988 [astro-ph.CO]].

[27] D. S. Akerib et al. [LUX Collaboration], Phys. Rev. Lett. 112, 091303 (2014) [arXiv:1310.8214 [astro-ph.CO]].

[28] D. C. Malling, D. S. Akerib, H. M. Araujo, X. Bai, S. Bedikian, E. Bernard, A. Bernstein and A. Bradley et al., arXiv:1110.0103 [astro-ph.IM].

[29] J. Billard, L. Strigari and E. Figueroa-Feliciano, Phys. Rev. D 89, no. 2, 023524 (2014) [arXiv:1307.5458 [hep-ph]];

[30] P. Cushman et al., arXiv:1310.8327 [hep-ex].

[31] O. Buchmueller et al., Eur. Phys. J. C 72, 1878 (2012) doi:10.1140/epjc/s10052-012-1878-4 [arXiv:1110.3568 [hep-ph]]; O. Buchmueller, et al., Eur. Phys. J. C 72 (2012) 2020 [arXiv:1112.3564 [hep-ph]]. H. Baer, V. Barger and A. Mustafayev, JHEP 1205 (2012) 091 [arXiv:1202.4038 [hep-ph]]; P. Bechtle, T. Bringmann, K. Desch, H. Dreiner, M. Hamer, C. Hensel, M. Kramer and N. Nguyen et al., JHEP 1206, 098 (2012) [arXiv:1204.4199 [hep-ph]]; A. Fowlie, M. Kazana, K. Kowalska, S. Munir, L. Roszkowski, E. M. Sessolo, S. Trojanowski and Y. L. S. Tsai, Phys. Rev. D 86, 075010 (2012) doi:10.1103/PhysRevD.86.075010 [arXiv:1206.0264 [hep-ph]]; T. Li, J. A. Maxin,

D. V. Nanopoulos and J. W. Walker, Europhys. Lett. 100, 21001 (2012) doi:10.1209/0295-5075/100/21001 [arXiv:1206.2633 [hep-ph]]; K. Kowalska et al. [BayesFITS Group Collaboration], Phys. Rev. D 87, no. 11, 115010 (2013) [arXiv:1211.1693 [hep-ph]]; C. Strege, G. Bertone, F. Feroz, M. Fornasa, R. Ruiz de Austri and R. Trotta, JCAP 1304, 013 (2013) doi:10.1088/1475-7516/2013/04/013 [arXiv:1212.2636 [hep-ph]]; T. Cohen and J. G. Wacker, JHEP 1309 (2013) 061 [arXiv:1305.2914 [hep-ph]]; S. Henrot-Versillé, Rém. Lafaye, T. Plehn, M. Rauch, D. Zerwas, S. ép. Plaszczynski, B. Rouillé d'Orfeuil and M. Spinelli, Phys. Rev. D 89, 055017 (2014) [arXiv:1309.6958 [hep-ph]]; P. Bechtle et al., PoS EPS -HEP2013, 313 (2013) [arXiv:1310.3045 [hep-ph]]; L. Roszkowski, E. M. Sessolo and A. J. Williams, JHEP 1408, 067 (2014) [arXiv:1405.4289 [hep-ph]]; P. Bechtle et al., Eur. Phys. J. C 76, no. 2, 96 (2016) doi:10.1140/epjc/s10052-015-3864-0 [arXiv:1508.05951 [hep-ph]].

[32] G. Aad et al. [ATLAS Collaboration], JHEP 1409 (2014) 176 [arXiv:1405.7875 [hep-ex]]; JHEP 1510, 054 (2015) doi:10.1007/JHEP10(2015)054 [arXiv:1507.05525 [hep-ex]]; full ATLAS Run 1 results can be found at https://twiki.cern.ch/twiki/bin/view/AtlasPublic/SupersymmetryPublicResults.

[33] N. Arkani-Hamed, A. Delgado and G. F. Giudice, Nucl. Phys. B 741 (2006) 108 [hep-ph/0601041].

[34] O. Buchmueller et al., Eur. Phys. J. C 74 (2014) 2922 [arXiv:1312.5250 [hep-ph]].

[35] L. Baudis [DARWIN Consortium Collaboration], J. Phys. Conf. Ser. 375 (2012) 012028 [arXiv:1201.2402 [astro-ph.IM]]. 
[36] J. Ellis, T. Falk, and K.A. Olive, Phys. Lett. B444 (1998) 367 [arXiv:hep-ph/9810360]; J. Ellis, T. Falk, K.A. Olive, and M. Srednicki, Astr. Part. Phys. 13 (2000) 181 [Erratum-ibid. 15 (2001) 413] [arXiv:hep-ph/9905481]; R. Arnowitt, B. Dutta and Y. Santoso, Nucl. Phys. B 606 (2001) 59 [arXiv:hep-ph/0102181]; M. E. Gómez, G. Lazarides and C. Pallis, Phys. Rev. D D61 (2000) 123512 [arXiv:hep-ph/9907261]; Phys. Lett. B487 (2000) 313 [arXiv:hep-ph/0004028]; Nucl. Phys. B B638 (2002) 165 [arXiv:hep-ph/0203131]; T. Nihei, L. Roszkowski and R. Ruiz de Austri, JHEP 0207 (2002) 024 [arXiv:hep-ph/0206266].

[37] M. Citron, J. Ellis, F. Luo, J. Marrouche, K. A. Olive and K. J. de Vries, Phys. Rev. D 87, no. 3, 036012 (2013) doi:10.1103/PhysRevD.87.036012 [arXiv:1212.2886 [hep-ph]].

[38] T. Hahn, S. Heinemeyer, W. Hollik, H. Rzehak and G. Weiglein, Phys. Rev. Lett. 112, no. 14, 141801 (2014) doi:10.1103/PhysRevLett.112.141801 [arXiv:1312.4937 [hep-ph]].

[39] O. Buchmueller, M. Citron, J. Ellis, S. Guha, J. Marrouche, K. A. Olive, K. de Vries and J. Zheng, Eur. Phys. J. C 75, no. 10, 469 (2015) doi:10.1140/epjc/s10052-015-3675-3 [arXiv:1505.04702 [hep-ph]].

[40] ATLAS Collaboration, http://cdsweb.cern.ch/record/1472710.

[41] G. Aad et al. [ATLAS Collaboration], Phys. Lett. B 720, 277 (2013) doi:10.1016/j.physletb.2013.02.015 [arXiv:1211.1597 [hep-ex]].

[42] G. Aad et al. [ATLAS Collaboration], Phys. Lett. B 716, 1 (2012) [arXiv:1207.7214 [hep-ex]]; S. Chatrchyan et al. [CMS Collaboration], Phys. Lett. B 716, 30 (2012) [arXiv:1207.7235 [hep-ex]]; G. Aad et al. [ATLAS and CMS Collaborations], Phys. Rev. Lett. 114 (2015) 191803 [arXiv:1503.07589 [hep-ex]].

[43] C. Boehm, A. Djouadi and M. Drees, Phys. Rev. D 62, 035012 (2000) [arXiv:hep-ph/9911496]; J. R. Ellis, K. A. Olive and Y. Santoso, Astropart. Phys. 18, 395 (2003) [arXiv:hep-ph/0112113]; J. Edsjo, M. Schelke, P. Ullio and P. Gondolo, JCAP 0304, 001 (2003) [arXiv:hep-ph/0301106]; J. L. Diaz-Cruz, J. R. Ellis, K. A. Olive and Y. Santoso, JHEP 0705, 003 (2007) [arXiv:hep-ph/0701229]; I. Gogoladze, S. Raza and Q. Shafi, Phys. Lett. B 706, 345 (2012) [arXiv:1104.3566 [hep-ph]]; M. A. Ajaib, T. Li and Q. Shafi, Phys. Rev. D 85, 055021 (2012) [arXiv:1111.4467 [hep-ph]]; J. Harz, B. Herrmann, M. Klasen, K. Kovarik and Q. L. Boulc'h, Phys. Rev. D 87 (2013) 5, 054031 [arXiv:1212.5241]; J. Harz, B. Herrmann, M. Klasen and K. Kovarik, Phys. Rev. D 91 (2015) 3, 034028 [arXiv:1409.2898 [hep-ph]]; A. Ibarra, A. Pierce, N. R. Shah and S. Vogl, Phys. Rev. D 91, no. 9, 095018 (2015) [arXiv:1501.03164 [hep-ph]].

[44] J. Ellis, K. A. Olive and J. Zheng, Eur. Phys. J. C 74 (2014) 2947 [arXiv:1404.5571 [hep-ph]].

[45] S. Raza, Q. Shafi and C. S. Ün, Phys. Rev. D 92, no. 5, 055010 (2015) [arXiv:1412.7672 [hep-ph]].

[46] P. A. R. Ade et al. [Planck Collaboration], arXiv:1502.01589 [astro-ph.CO].

[47] K. A. Olive and M. Srednicki, Phys. Lett. B 230, 78 (1989); K. A. Olive and M. Srednicki, Nucl. Phys. B 355, 208 (1991).

[48] S. Profumo and C. E. Yaguna, Phys. Rev. D 69, 115009 (2004) [hep-ph/0402208]; D. Feldman, Z. Liu and P. Nath, Phys. Rev. D 80, 015007 (2009) [arXiv:0905.1148 [hep-ph]]; N. Chen, D. Feldman, Z. Liu, P. Nath and G. Peim, Phys. Rev. D 83, 035005 (2011) [arXiv:1011.1246 [hep-ph]]; I. Gogoladze, R. Khalid and Q. Shafi, Phys. Rev. D 79, 115004 (2009) [arXiv:0903.5204 [hep-ph]]; I. Gogoladze, R. Khalid and Q. Shafi, Phys. Rev. D 80, 095016 (2009) [arXiv:0908.0731 [hep-ph]]; M. Adeel Ajaib, T. Li, Q. Shafi and K. Wang, JHEP 1101, 028 (2011) [arXiv:1011.5518 [hep-ph]]; K. Harigaya, M. Ibe and T. T. Yanagida, JHEP 1312, 016 (2013) [arXiv:1310.0643 [hep-ph]]; 
K. Harigaya, K. Kaneta and S. Matsumoto, Phys. Rev. D 89, no. 11, 115021 (2014) [arXiv:1403.0715 [hep-ph]]; J. L. Evans and K. A. Olive, Phys. Rev. D 90, no. 11, 115020 (2014) [arXiv:1408.5102 [hep-ph]]; A. De Simone, G. F. Giudice and A. Strumia, JHEP 1406, 081 (2014) [arXiv:1402.6287 [hep-ph]]; M. Low and L. T. Wang, JHEP 1408, 161 (2014) [arXiv:1404.0682 [hep-ph]].

[49] J. Ellis, F. Luo and K. A. Olive, JHEP 1509, 127 (2015) [arXiv:1503.07142 [hep-ph]].

[50] J. Ellis, J. L. Evans, F. Luo and K. A. Olive, JHEP 1602, 071 (2016) doi:10.1007/JHEP02(2016)071 [arXiv:1510.03498 [hep-ph]].

[51] R.Aaij et al. [LHCb Collaboration], Phys. Rev. Lett. 111 (2013) 101805 [arXiv:1307.5024 [hep-ex]]; S. Chatrchyan et al. [CMS Collaboration], Phys. Rev. Lett. 111 (2013) 101804 [arXiv:1307.5025 [hep-ex]]; R.Aaij et al. [LHCb and CMS Collaborations], LHCb-CONF-2013-012, CMS PAS BPH-13-007; V. Khachatryan et al. [CMS and LHCb Collaborations], Nature 522, 68 (2015) [arXiv:1411.4413 [hep-ex]].

[52] H. Baer, A. Mustafayev, S. Profumo, A. Belyaev and X. Tata, Phys. Rev. D 71, 095008 (2005) [arXiv:hep-ph/0412059]; H. Baer, A. Mustafayev, S. Profumo, A. Belyaev and X. Tata, JHEP 0507 (2005) 065, hep-ph/0504001.

[53] J. R. Ellis, K. A. Olive and P. Sandick, Phys. Rev. D 78, 075012 (2008) [arXiv:0805.2343 [hep-ph]].

[54] J. Ellis, K. Olive and Y. Santoso, Phys. Lett. B 539, 107 (2002) [arXiv:hep-ph/0204192]; J. R. Ellis, T. Falk, K. A. Olive and Y. Santoso, Nucl. Phys. B 652, 259 (2003) [arXiv:hep-ph/0210205].

[55] J. R. Ellis, K. A. Olive and P. Sandick, Phys. Lett. B 642, 389 (2006) [hep-ph/0607002]; J. R. Ellis, K. A. Olive and P. Sandick, JHEP 0706, 079 (2007) [arXiv:0704.3446 [hep-ph]]; J. R. Ellis, K. A. Olive and P. Sandick, JHEP 0808, 013 (2008) [arXiv:0801.1651 [hep-ph]].

[56] L. Calibbi, Y. Mambrini and S. K. Vempati, JHEP 0709, 081 (2007) [arXiv:0704.3518 [hep-ph]]; L. Calibbi, A. Faccia, A. Masiero and S. K. Vempati, Phys. Rev. D 74, 116002 (2006) [arXiv:hep-ph/0605139]; E. Carquin, J. Ellis, M. E. Gomez, S. Lola and J. Rodriguez-Quintero, JHEP 0905 (2009) 026 [arXiv:0812.4243 [hep-ph]]; J. Ellis, A. Mustafayev and K. A. Olive, Eur. Phys. J. C 69, 201 (2010) [arXiv:1003.3677 [hep-ph]]; J. Ellis, A. Mustafayev and K. A. Olive, Eur. Phys. J. C 69, 219 (2010) [arXiv:1004.5399 [hep-ph]]; J. Ellis, A. Mustafayev and K. A. Olive, Eur. Phys. J. C 71, 1689 (2011) [arXiv:1103.5140 [hep-ph]]; E. Dudas, Y. Mambrini, A. Mustafayev and K. A. Olive, Eur. Phys. J. C 72, 2138 (2012) [Eur. Phys. J. C 73, 2430 (2013)] [arXiv:1205.5988 [hep-ph]]; E. Dudas, A. Linde, Y. Mambrini, A. Mustafayev and K. A. Olive, Eur. Phys. J. C 73, no. 1, 2268 (2013) [arXiv:1209.0499 [hep-ph]].

[57] R. Barbieri, S. Ferrara and C. A. Savoy, Phys. Lett. B 119, 343 (1982).

[58] J. R. Ellis, K. A. Olive, Y. Santoso and V. C. Spanos, Phys. Lett. B 573 (2003) 162 [arXiv:hep-ph/0305212], and Phys. Rev. D 70 (2004) 055005 [arXiv:hep-ph/0405110].

[59] M. Ibe, T. Moroi and T. T. Yanagida, Phys. Lett. B 644, 355 (2007) [hep-ph/0610277]; M. Ibe and T. T. Yanagida, Phys. Lett. B 709, 374 (2012) [arXiv:1112.2462 [hep-ph]]; M. Ibe, S. Matsumoto and T. T. Yanagida, Phys. Rev. D 85, 095011 (2012) [arXiv:1202.2253 [hep-ph]].

[60] M. Dine and D. MacIntire, Phys. Rev. D 46, 2594 (1992) [hep-ph/9205227]; L. Randall and R. Sundrum, Nucl. Phys. B 557, 79 (1999) [arXiv:hep-th/9810155]; G. F. Giudice, M. A. Luty, H. Murayama and R. Rattazzi, JHEP 9812, 027 (1998) [arXiv:hep-ph/9810442]; J. A. Bagger, T. Moroi and E. Poppitz, JHEP 0004, 009 (2000) [arXiv:hep-th/9911029]; P. Binetruy, M. K. Gaillard and B. D. Nelson, Nucl. Phys. B 604, 32 (2001) [arXiv:hep-ph/0011081]. 
[61] J. L. Evans, M. Ibe, K. A. Olive and T. T. Yanagida, Eur. Phys. J. C 73, 2468 (2013) [arXiv:1302.5346 [hep-ph]]; J. L. Evans, K. A. Olive, M. Ibe and T. T. Yanagida, Eur. Phys. J. C 73, 2611 (2013) [arXiv:1305.7461 [hep-ph]]; J. L. Evans, M. Ibe, K. A. Olive and T. T. Yanagida, Phys. Rev. D 91, 055008 (2015) doi:10.1103/PhysRevD.91.055008 [arXiv:1412.3403 [hep-ph]]; J. L. Evans, N. Nagata and K. A. Olive, Phys. Rev. D 91, 055027 (2015) doi:10.1103/PhysRevD.91.055027 [arXiv:1502.00034 [hep-ph]].

[62] J. D. Wells, hep-ph/0306127; N. Arkani-Hamed and S. Dimopoulos, JHEP 0506, 073 (2005) [arXiv:hep-th/0405159]; G. F. Giudice and A. Romanino, Nucl. Phys. B 699, 65 (2004) [Erratum-ibid. B 706, 65 (2005)] [arXiv:hep-ph/0406088]; N. Arkani-Hamed, S. Dimopoulos, G. F. Giudice and A. Romanino, Nucl. Phys. B 709, 3 (2005) [arXiv:hep-ph/0409232]; J. D. Wells, Phys. Rev. D 71, 015013 (2005) [arXiv:hep-ph/0411041].

[63] John R. Ellis, S. Kelley and Dimitri V. Nanopoulos, Phys. Lett. B 249, 441, 1990; John R. Ellis, S. Kelley and Dimitri V. Nanopoulos, Phys. Lett. B 260, 131, 1991; Ugo Amaldi, Wim de Boer, and Hermann Furstenau. Phys. Lett., B 260, 447, 1991; Paul Langacker and Ming-xing Luo, Phys. Rev. D 44, 817, 1991; C. Giunti, C. W. Kim and U. W. Lee, Mod. Phys. Lett. A 6, 1745, 1991.

[64] John R. Ellis and Douglas Ross, Phys. Lett. B 506, 331, 2001, hep-ph/0012067.

[65] L. E. Ibanez and G. G. Ross, Phys. Lett. B 110, 215 (1982); K. Inoue, A. Kakuto, H. Komatsu and S. Takeshita, Prog. Theor. Phys. 68, 927 (1982) [Erratum-ibid. 70, 330 (1983)] [Prog. Theor. Phys. 70, 330 (1983)]; L. E. Ibanez, Phys. Lett. B 118, 73 (1982); J. R. Ellis, D. V. Nanopoulos and K. Tamvakis, Phys. Lett. B 121, 123 (1983); J. R. Ellis, J. S. Hagelin, D. V. Nanopoulos and K. Tamvakis, Phys. Lett. B 125, 275 (1983); L. Alvarez-Gaume, J. Polchinski and M. B. Wise, Nucl. Phys. B 221, 495 (1983).

[66] H. Goldberg, Phys. Rev. Lett. 50 (1983) 1419; J. Ellis, J. Hagelin, D. Nanopoulos, K. Olive and M. Srednicki, Nucl. Phys. B 238 (1984) 453.

[67] L. Maiani, in Proceedings, Gif-sur-Yvette Summer School On Particle Physics, 1979, 1-52; Gerard 't Hooft and others (eds.), Recent Developments in Gauge Theories, Proceedings of the Nato Advanced Study Institute, Cargese, France, August 26 - September 8, 1979, Plenum press, New York, USA, 1980, Nato Advanced Study Institutes Series: Series B, Physics, 59.; Edward Witten, Phys. Lett. B105, 267, 1981.

[68] R. Slansky, Phys. Rept. 79, 1 (1981).

[69] M. Shiozawa, talk presented at TAUP 2013, September 8-13, Asilomar, CA, USA.

[70] K. S. Babu, E. Kearns, U. Al-Binni, S. Banerjee, D. V. Baxter, Z. Berezhiani, M. Bergevin and S. Bhattacharya et al., arXiv:1311.5285 [hep-ph].

[71] V. Silveira and A. Zee, Phys. Lett. B 161, 136 (1985); J. McDonald, Phys. Rev. D 50, 3637 (1994) [hep-ph/0702143]; C. P. Burgess, M. Pospelov and T. ter Veldhuis, Nucl. Phys. B 619, 709 (2001) [hep-ph/0011335]; H. Davoudiasl, R. Kitano, T. Li and H. Murayama, Phys. Lett. B 609, 117 (2005) [hep-ph/0405097].

[72] N. G. Deshpande and E. Ma, Phys. Rev. D 18, 2574 (1978); E. Ma, Phys. Rev. D 73, 077301 (2006) [hep-ph/0601225]; R. Barbieri, L. J. Hall and V. S. Rychkov, Phys. Rev. D 74, 015007 (2006) [hep-ph/0603188]; L. Lopez Honorez, E. Nezri, J. F. Oliver and M. H. G. Tytgat, JCAP 0702, 028 (2007) [hep-ph/0612275].

[73] A. Arhrib, Y. L. S. Tsai, Q. Yuan and T. C. Yuan, JCAP 1406, 030 (2014) [arXiv:1310.0358 [hep-ph]]; A. Ilnicka, M. Krawczyk and T. Robens, Phys. Rev. D 93, no. 5, 055026 (2016) doi:10.1103/PhysRevD.93.055026 [arXiv:1508.01671 [hep-ph]]. 